\title{
Choroidal Modifications Preceding the Onset of Macular Neovascularization in Age-Related Macular Degeneration
}

\author{
Alessandro Arrigo (D) - Alessia Amato · Costanza Barresi · \\ Emanuela Aragona · Andrea Saladino · Adelaide Pina • \\ Francesca Calcagno · Francesco Bandello • Maurizio Battaglia Parodi
}

Received: October 30, 2021 / Accepted: December 2, 2021 / Published online: December 19, 2021

(C) The Author(s) 2021

\section{ABSTRACT}

Introduction: Macular neovascularization (MNV) is a common complication of age-related macular degeneration (AMD). Although several biomarkers may help to estimate the risk of MNV onset, neovascular complication is difficult to predict. Previous studies showed that the quantitative assessment of choroidal and choriocapillaris changes is useful for the assessment of atrophy expansion. On the other hand, scant data are available regarding the role of this kind of assessment in the setting of MNV. The aim of the study is to analyze choroidal and choriocapillaris changes occurring before the onset of MNV in patients affected by AMD using quantitative optical coherence tomography (OCT) and OCT angiography (OCTA).

Methods: The study was designed as a retrospective case series. Patients affected by AMD, categorized in eyes complicated by MNV and eyes not developing MNV, were retrospectively analyzed for 1 year of follow-up. Choroidal thickness (CT), Sattler layer thickness (SLT) and Haller layer thickness (HLT) were measured on OCT scans. Vessel density (VD) and

A. Arrigo $(\varangle) \cdot$ A. Amato - C. Barresi - E. Aragona · A. Saladino - A. Pina - F. Calcagno - F. Bandello . M. Battaglia Parodi Department of Ophthalmology, IRCCS San Raffaele Scientific Institute, via Olgettina 60, 20132 Milan, Italy

e-mail: alessandro.arrigo@hotmail.com choriocapillaris (CC) porosity were quantified on OCTA reconstructions. The main outcome measure was the relationship between choroidal and CC parameters, and MNV onset.

Results: We included 50 eyes of 50 AMD patients ( 28 male; mean age $74 \pm 5$ years). Over the 1-year follow-up, 15/50 eyes developed MNV (9 type $1 ; 3$ type 2; 3 mixed type $1-2$ ). Mean best-corrected visual acuity (BCVA) was $0.15 \pm 0.15 \log$ MAR at baseline, remaining stable in eyes not developing $\mathrm{MNV}(0.15 \pm 0.12$ $\log$ MAR; $p>0.05)$, and worsening to $0.38 \pm 0.20 \log$ MAR in eyes developing $\mathrm{MNV}$ $(p<0.01)$. VD values were similar between eyes developing MNV and eyes not complicated by MNV at baseline, with significant worsening detected only in MNV eyes. CC porosity was significantly higher in MNV eyes already before the onset of MNV. Furthermore, SLT was significantly lower in eyes developing MNV. The onset of MNV was preceded by a significant increase in intraretinal hyperreflective foci, whereas choroidal hyperreflective foci showed no evident changes.

Conclusions: The degeneration of CC and the SLT thinning represent early an biomarker of MNV onset in AMD.

Keywords: AMD; Choriocapillaris; Choroid; MNV; OCT; OCTA 


\section{Key Summary Points}

Onset of macular neovascularization (MNV) is associated with the impairment of the choriocapillaris/choroidal complex.

Choriocapillaris porosity represents a quantitative biomarker associated with age-related macular degeneration (AMD) progression.

The meaningful choroidal modification is represented by the thinning of the Sattler layer.

Quantitative optical coherence tomography angiography (OCTA)-based approaches may provide clinically relevant cutoffs to optimize the management of AMD patients.

\section{INTRODUCTION}

The onset of macular neovascularization (MNV) in age-related macular degeneration (AMD) is regulated by several metabolic factors and cytokines [1], whose release is promoted by the progressive distress of the retinal pigment epithelium (RPE). The contribution of the choroid and, more specifically, the choriocapillaris in the pathogenesis of AMD and in its progression to the advanced stages of the disease is debated. Both clinical and histologic studies demonstrated that choroidal modifications and vascular depletion are associated with AMD onset and progression towards both dry and wet forms $[2,3]$. However, although several studies have highlighted its role in the onset and progression of geographic atrophy [4-7], data regarding its relationship with the onset of MNV are scant.

The main aim of the present study is to assess the role of quantitative optical coherence tomography (OCT) angiography (OCTA) in assessing the alterations occurring at the choroidal level before the development of the MNV.

\section{METHODS}

The study was designed as a retrospective case series. Fifty patients affected by intermediate AMD were recruited at the Department of Ophthalmology of IRCCS San Raffaele Hospital, Milan, Italy. We included patients with a diagnosis of intermediate AMD and at least 1 year of follow-up before the onset of MNV. Exclusion criteria were a diagnosis of other ocular diseases (glaucoma or other optic nerve diseases, uveitis, diabetic retinopathy or other vascular disorders), ophthalmic surgery in the last 6 months before the inclusion, high media opacities, and systemic diseases potentially affecting the results of the study (uncontrolled arterial hypertension, uncontrolled diabetes mellitus, systemic drugs associated with retinal toxicity). We obtained signed informed consent from all the patients before the inclusion. The study was approved by the ethics committee of IRCCS Scientific Institute San Raffaele Hospital (MIRD), which verified its conduction in accordance with the Declaration of Helsinki.

\section{Data Acquisition}

We started from the last follow-up examination and retrospectively analyzed the 12-month (baseline) time point, both for eyes complicated by MNV and eyes not developing MNV. We considered only eyes with the intermediate AMD form maintained for at least one month since the baseline visit. In the case of the bilateral intermediate AMD form, the study eye was randomly selected. For inclusion in the study, patients were required to undergo complete clinical and imaging assessments over the entire follow-up window. We collected all the ophthalmological data, including best-corrected visual acuity (BCVA) measured by ETDRS chart, anterior and posterior slit-lamp examination, tonometry, optical coherence tomography (OCT) (HRA2+OCT Spectralis, Heidelberg Engineering, Germany) and OCT angiography (OCTA) (DRI OCT Triton, Topcon Corporation, Japan). The eyes complicated by MNV, confirmed by fluorescein angiography examination and classified in accordance with the Consensus 
on Neo-vascular AMD Nomenclature (CONAN) nomenclature [8], were treated by anti-VEGF intravitreal injections.

The OCT acquisition protocol was standardized for all the patients, including radial (automated real-time tracking $[$ ART $]=100$ frames, enhanced-depth imaging [EDI], 1536 A-scans, $30^{\circ}$ scan length, six sections), raster (ART $=30$, EDI, $1024 \mathrm{~A}$-scans, $20^{\circ} \times 15^{\circ}$ volume length, 19 sections, interline space $240 \mu \mathrm{m}$ ) and dense $\left(\mathrm{ART}=15, \mathrm{EDI}, 15^{\circ} \times 5^{\circ}\right.$ volume length, 49 sections, 384 A-scans, interline space $30 \mu \mathrm{m}$ ) acquisitions with ART 25 and EDI. The OCTA protocol included $3 \times 3 \mathrm{~mm}$ and $6 \times 6 \mathrm{~mm}$ scans. Only high-quality data, defined as Topcon Quality Index $>70$, were mandatorily included.

\section{Data Analysis}

Two independent expert graders (AA, EA) performed the manual measurements of choroidal thickness (CT), Haller layer thickness (HLT) and Sattler layer thickness (SLT) using Heidelberg segmentation tools on horizontal, foveal-centered, EDI radial scan. The extreme boundaries were defined as the lower margin of the RPE and the upper margin of the choroidal-scleral interface. CT and HLT were obtained from the mean value of five samples (subfoveal, $750 \mu \mathrm{m}$ [right-left] and $1500 \mu \mathrm{m}$ [right-left]), performed in a horizontal high-resolution OCT foveal scan, whereas SLT was considered as the result of (CT-HLT) in order to address the difficulty to properly detect SLT boundaries.

OCTA reconstructions of the superficial capillary plexus (SCP), deep capillary plexus (DCP) and choriocapillaris (CC) were obtained from the automatic segmentations of Topcon software, manually corrected by the same two independent expert graders to minimize potential artifacts. Vessel density (VD) was calculated through in-house scripts used in ImageJ software [9]. For the statistical analyses, we considered baseline and 12-month measurements.

Moreover, we calculated CC porosity, intended as a novel parameter for the measurement of flow voids in the CC. To calculate CC porosity, we started from OCTA CC binarized reconstructions and adopted the Image porosity pipeline to measure the percentage CC porosity [10]. Before calculating the percentages of CC flow voids, we performed the segmentation of drusen with a mean diameter $>75 \mu \mathrm{m}$ [11], guided by OCT images, and we also performed the segmentation of retinal vessels, considered as projection artifacts, and of MNV area (MNV network + perilesional fluid area). All these segmentations were considered as exclusion criteria for CC porosity calculation.

We also evaluated intraretinal and choroidal hyperreflective foci (HF) changes occurring before the onset of MNV. These have been defined as well-circumscribed, discrete, dotshaped, hyperreflective lesions. In detail, we used high-resolution OCT scans with EDI, inverted white-black colors to better highlight $\mathrm{HF}$, and centered the scans on the region developing MNV. To make the analysis and interpretation of $\mathrm{HF}$ easier and more reproducible, we defined OCT scans showing at least $\mathrm{HF}>5$ in the sector involved in MNV development as positive for HF. Furthermore, we arbitrarily considered scans showing an increase of $\mathrm{HF}>5$, compared with the previous followup, as positive for an increasing number of HF. The absolute value of HF was used to perform the correlation analyses with all the other parameters. The follow-up tool provided by Heidelberg software was adopted to perform the analysis of HF number changes in the same retinal scans.

All these measurements were taken at least twice on the same scans to calculate repeatability and reproducibility. The interclass correlation coefficient (ICC) was calculated as well, in order to assess inter-grader agreement through a two-way random-effects model.

The main outcome measure was the relationship between choroidal and CC parameters and the development of MNV. The secondary outcome was the correlation with intraretinal and choroidal HF modifications. 


\section{Statistical Analysis}

Age, gender and concomitant diseases were considered as fixed factors for the statistical analysis. We used a linear mixed model to evaluate the normality distribution of each variable, assessed through frequency histograms and quantile-quantile plots. Descriptive statistics of continuous variables were reported as mean $\pm \mathrm{SD}$; frequency and proportions were described as categorical variables. Continuous variables were analyzed by two-tailed $t$ test. Further analyses were conducted separately analyzing eyes developing MNV and eyes not developing neovascular complications. TauKendall correlation analysis was adopted to assess the statistical relationship among all the variables considered. In view of the multiple testing, the Bonferroni approach was considered the most appropriate to address the question of multiple comparisons. The statistical significance threshold was set considering the presence of 10 quantitative variables (BCVA, CT, HLT, SLT, SCP VD, DCP VD, CC VD, CC porosity, intraretinal $\mathrm{HF}$, choroidal $\mathrm{HF}$ ), making it necessary to reach a $p$ value $\leq 0.005(0.05 /$ $10)$. The statistical analysis was performed by means of an SPSS software package (SPSS, Chicago, Illinois, USA).

Table 1 Baseline features. The following abbreviations are used: best corrected visual acuity (BCVA), central macular thickness (CMT), macular neovascularization (MNV)

\begin{tabular}{ll}
\hline Parameter & AMD patients \\
\hline No. of patient (eyes) & $50(50)$ \\
Gender (M/F) & $28 / 22$ \\
Age (years) & $74 \pm 5$ \\
LogMAR BCVA & $0.15 \pm 0.15$ \\
CMT & $322 \pm 15$ \\
Fellow eye with MNV & $6 / 50$ \\
Pseudophakia & $50 / 50$ \\
\hline
\end{tabular}

\section{RESULTS}

\section{Demographic and Clinical Data}

Fifty eyes of 50 AMD patients (28 males; mean age $74 \pm 5$ years) were included in the study (Table 1). Over the 1-year follow-up, 15/50 eyes developed MNV (9 type 1; 3 type 2; 3 mixed type 1-2). In detail, $8 / 15$ eyes belonged to patients with neovascular AMD in the fellow eye, whereas in $6 / 15$ eyes the contralateral eye was affected by intermediate AMD $(p>0.05)$.

Mean BCVA was $0.15 \pm 0.15 \log$ MAR at baseline and found to be stable in eyes not developing $\mathrm{MNV} \quad(0.15 \pm 0.12 \quad \log \mathrm{MAR}$; $p>0.05)$, worsening to $0.38 \pm 0.20 \log$ MAR in eyes developing MNV $(p<0.005)$.

OCTA quantitative analysis showed similar VD values for all the plexa considered at baseline (all $p>0.05$ ). On the other hand, DCP and CC VD values proved significantly worse in the MNV group than in the no-MNV group $(p<0.005)$ (Table 2).

\section{Quantitative Imaging Data}

The quantitative evaluation of CC porosity revealed that although it was similar between the two groups at baseline, it had worsened in eyes developing MNV by the 12-month evaluation $(p<0.005)$ (Fig. 1).

The evaluation of the choroid revealed comparable CT and HLT between groups both at baseline and at the 1-year follow-up. Nevertheless, SLT was found to be significantly lower in eyes developing MNV both at baseline and at the 1-year follow-up, compared with eyes not developing MNV ( $p<0.005)$ (Table 2$)$.

Degeneration of CC porosity was correlated with SLT thinning, as confirmed by the statistically significant correlation (Tau-Kendall correlation coeff. $-0.59 ; p<0.005)$, whereas no significant correlations were found among the other OCTA quantitative parameters evaluated $(p>0.05)$.

The evaluation of intraretinal and choroidal HF showed that all the $15 / 15$ eyes (100\%) developing MNV were affected by a significant and progressive increase in intraretinal $\mathrm{HF}$ 
Table 2 OCT and OCTA quantitative data. All the variables are expressed as MEAN $\pm S D$

\begin{tabular}{|c|c|c|c|}
\hline \multicolumn{4}{|c|}{ OCT and OCTA quantitative MNV data } \\
\hline Parameter & MNV group & No-MNV group & $p$ Value \\
\hline CT baseline $(\mu \mathrm{m})$ & $216 \pm 78$ & $242 \pm 81$ & $p>0.05$ \\
\hline CT 1-year $(\mu \mathrm{m})$ & $201 \pm 85$ & $218 \pm 66$ & $p>0.05$ \\
\hline$p$ Value & $p>0.05$ & $p>0.05$ & \\
\hline HLT baseline $(\mu \mathrm{m})$ & $178 \pm 75$ & $190 \pm 76$ & $p=0.03$ \\
\hline HLT 1-year $(\mu \mathrm{m})$ & $166 \pm 76$ & $186 \pm 72$ & $p=0.03$ \\
\hline$p$ Value & $p=0.04$ & $p>0.05$ & \\
\hline SLT baseline $(\mu \mathrm{m})$ & $32 \pm 15$ & $61 \pm 25$ & $p<0.01^{*}$ \\
\hline SLT 1-year $(\mu \mathrm{m})$ & $22 \pm 13$ & $48 \pm 22$ & $p<0.01^{*}$ \\
\hline$p$ Value & $p<0.01^{*}$ & $p<0.01^{*}$ & \\
\hline VD SCP baseline & $0.37 \pm 0.02$ & $0.38 \pm 0.03$ & $p>0.05$ \\
\hline VD SCP 1-year & $0.36 \pm 0.04$ & $0.37 \pm 0.02$ & $p>0.05$ \\
\hline$p$ Value & $p>0.05$ & $p>0.05$ & \\
\hline VD DCP baseline & $0.34 \pm 0.01$ & $0.34 \pm 0.02$ & $p>0.05$ \\
\hline VD DCP 1-year & $0.28 \pm 0.02$ & $0.33 \pm 0.02$ & $p<0.01^{*}$ \\
\hline$p$ Value & $p<0.01^{*}$ & $p>0.05$ & \\
\hline VD CC baseline & $0.46 \pm 0.02$ & $0.47 \pm 0.03$ & $p>0.05$ \\
\hline VD CC 1-year & $0.42 \pm 0.02$ & $0.46 \pm 0.03$ & $p<0.01^{*}$ \\
\hline$p$ Value & $p<0.01^{*}$ & $p>0.05$ & \\
\hline CC porosity baseline (\%) & $9.8 \pm 0.3$ & $9.2 \pm 0.3$ & $p>0.05$ \\
\hline CC porosity 1-year (\%) & $17.3 \pm 0.3$ & $11.9 \pm 0.2$ & $p<0.01^{*}$ \\
\hline$p$ Value (baseline vs. 1-year) & $p<0.01^{*}$ & $p>0.05$ & \\
\hline
\end{tabular}

Statistically significant values are marked by asterisks $\left({ }^{*}\right)$. The following abbreviations are used: macular neovascularization $(M N V)$, choroidal thickness $(C T)$, Haller layer thickness $(H L T)$, Sattler layer thickness $(S L T)$, vessel density $(V D)$, superficial capillary plexus $(S C \mathrm{P})$, deep capillary plexus $(D C P)$, choriocapillaris $(C C)$

(Fig. 2). In contrast, eyes not developing MNV showed no HF variation. Furthermore, we did not observe any significant modifications in choroidal HF over the entire follow-up. The increased number of intraretinal HF significantly correlated both with CC porosity and SLT (Tau-Kendall correlation coeff. -0.53 and -0.52 , respectively; both $p<0.005$ ).

Overall, repeatability and reproducibility were 0.89 (range 0.87-0.92) and 0.89 (range
0.86-0.91), respectively $(p<0.005)$, and ICC was 0.90 (range $0.88-0.92 ; p<0.005$ ).

\section{DISCUSSION}

In the present study, we assessed the relationship between vascular impairment of the choroid and onset of MNV in patients affected by AMD. Our data showed that SLT was 


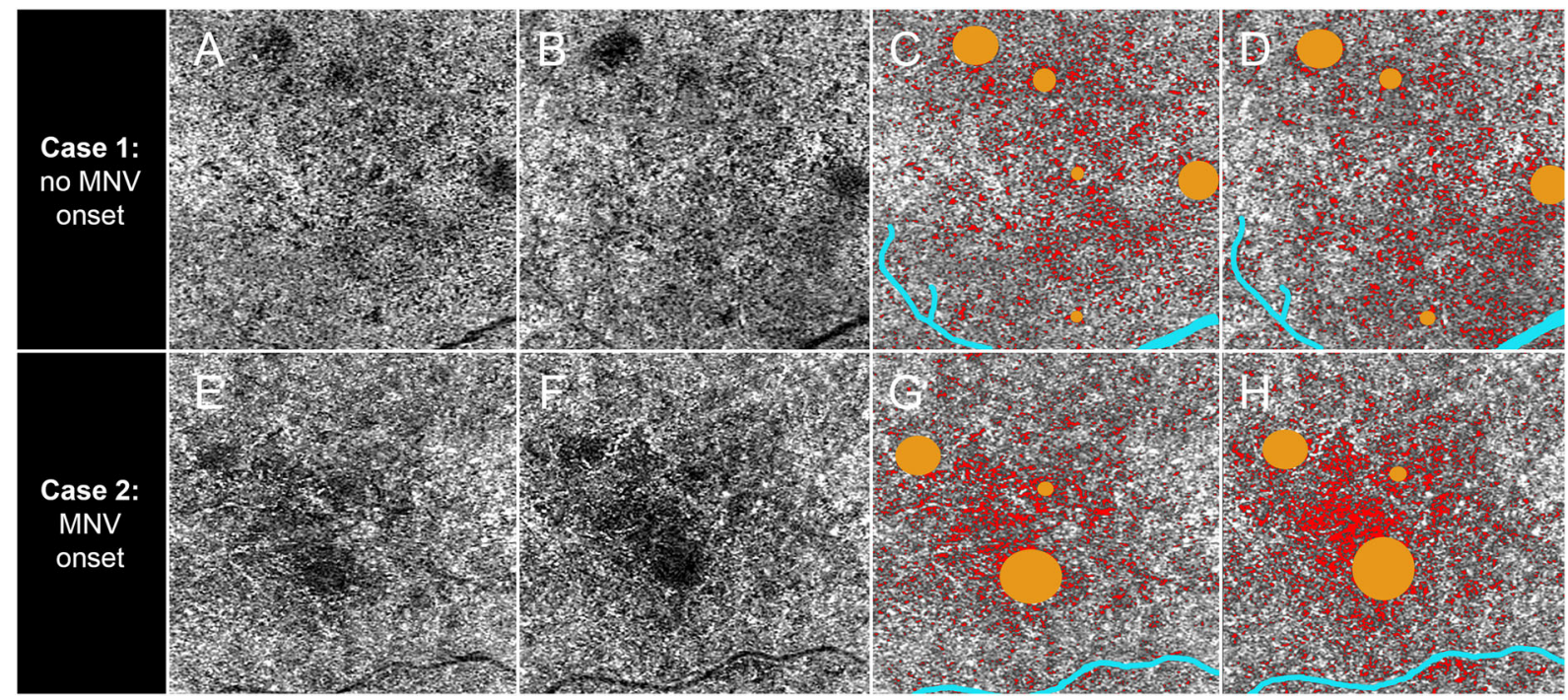

Fig. 1 Choriocapillaris porosity in AMD. The first case shows poor and sparse CC flow voids, together with masking phenomena caused by drusen at baseline (a). After one year, the CC status appears almost stable (b). The quantitative analysis shows comparable CC porosity profiles at baseline (c) and at the 12-month follow-up (d). This case did not develop MNV. The second case shows higher CC flow voids already at baseline (e), increasing considerably by the 1-year follow-up (f). The

significantly thinner in eyes developing MNV than in eyes not displaying this complication. Furthermore, OCTA analysis revealed an increase in CC porosity associated with $\mathrm{MNV}$ onset. Indeed, CC porosity was found to have increased significantly by the 12 -month examination in eyes developing MNV. On the other hand, CC porosity did not vary greatly over the 12-month follow-up in eyes not developing MNV. VD analyses showed minor changes in the retinal vascular network and in CC occurring over the follow-up.

The role of choroidal thinning has already been widely discussed in the context of the onset and progression of geographic atrophy, providing strong support for accurate choroidal assessment [2-7]. In contrast, data regarding choroidal impairment in the context of MNV complications are scant. A recent study described choroidal impairment in MNV eyes, as shown by significant modifications of both CT and choroidal vascularity index [12]. Our data are in line with these previous findings and quantitative analysis shows a remarkable increase in CC porosity, comparing baseline (g) and 1-year follow-up (h) images. This case turned out to be complicated by the onset of MNV. For both cases, CC porosity is shown in red, drusen are schematically localized in orange and vessel projections are marked in blue

provide new information regarding the different involvement of SLT and HLT, together with an OCTA-based evaluation of CC impairment. Furthermore, in our study we assessed the changes in intraretinal and choroidal HF occurring before the onset of MNV. Our data showed that intraretinal HF progressively increased before and during the development of MNV, whereas choroidal HF did not undergo very significant changes. This finding is in line with previous evidence suggesting a main intraretinal involvement in eyes heading towards advanced stages of AMD [13], unlike eyes affected by MNV secondary to other conditions, i.e. angioid streaks, where the alterations have a pronounced effect on the choroid and Bruch Membrane [14, 15]. Our correlation analysis, proving to be statistically significant when comparing the intraretinal HF increase and the progression of vascular impairment (SLT and CC porosity), further reinforces the hypothesis of a primary impairment of the RPE, 


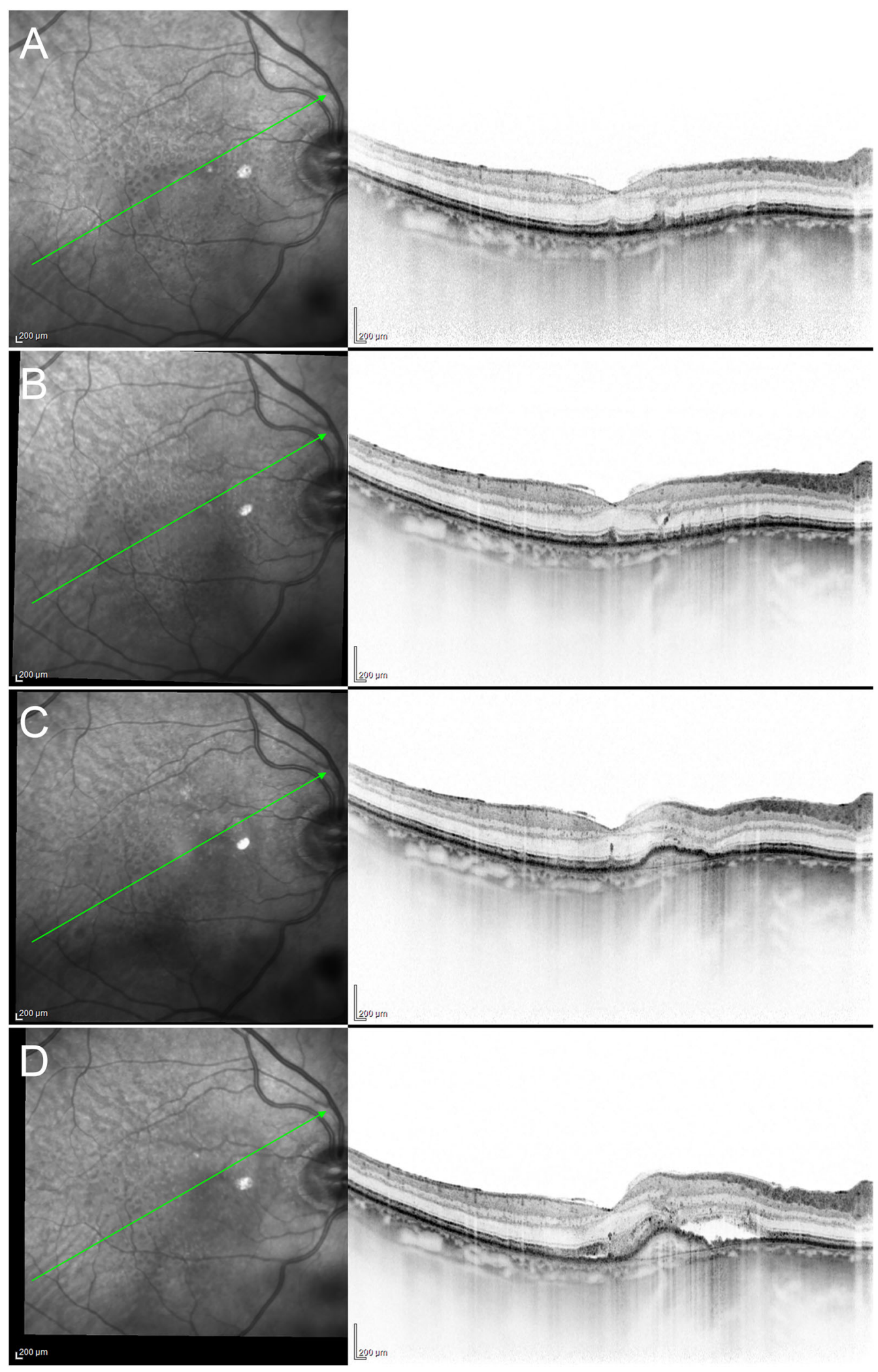


4Fig. 2 Hyperreflective foci modifications in eyes developing MNV. Baseline image, collected 12 months before the onset of $\mathrm{MNV}(\mathbf{a})$, showed unremarkable intraretinal and choroidal HF. The number of intraretinal HF progressively increased before the onset of MNV (2-month follow-up) (b), and further increased following the modifications leading to the MNV development (8-month and 10-month follow-up, respectively) (c, d, respectively). No significant modifications of choroidal HF were detected

prompting the later cascade of events leading to MNV development.

It is known that RPE and outer retinal distress is a major cause of increased production of VEGF and cytokines, leading to the onset of MNV [1]. The RPE/photoreceptor complex is mainly supported by the CC/choroidal vasculature [16]. It might be surmised that the microstructural mechanisms leading to the onset of the MNV are associated with the progressive occurrence of microcirculatory changes occurring at the level of the $\mathrm{CC} /$ choroidal complex. These may be detected before MNV onset by means of quantitative OCTA approaches, thus representing a potential early pathogenic biomarker associated with MNV development. This scenario is strongly supported by our findings, showing both SLT thinning and increasing CC porosity in eyes complicated by MNV. We may hypothesize this to be induced by a prior perfusion defect at the level of the Sattler layer, followed by a CC deterioration. The fact that CC porosity provided more evident signs of alterations, compared with CC VD, may be explained as the result of the VD parameter being less sensitive to initial alterations involving the CC plexus. Indeed, while VD mainly concerns the absolute percentage of pixels showing OCTA flow signal, CC porosity is mostly sensitive to the distribution of this signal, which may lead to an increased presence of CC flow voids in the presence of an uneven distribution of the CC blood flow perfusion.

We are aware that our study may labor under several limitations, first of all related to the relatively small number of eyes and the followup limited to 12-month. Our study quantitatively analyzed the reflectivity properties of MNV, but we are aware that there is yet no consensus about the best way to interpret this OCTA finding. Moreover, OCTA techniques are well-known to be potentially artifact prone, especially in the context of MNV development [17]. To reduce these potential influences, we segmented all the possible sources of CC masking effect, including retinal vessels, MNV, fluid and drusen, in order to exclude these regions from the analyses. Another important drawback is the lack of histopathological confirmation of MNV microstructural features. Furthermore, another limitation may be related to the retrospective design of the study; further prospective investigations with longer followup will be useful to draw more definite conclusions and to establish precise quantitative cutoffs related to MNV onset risk.

\section{CONCLUSIONS}

In conclusion, our study evaluated the role of SLT and CC porosity in assessing the risk of MNV onset. Albeit retaining the status of an initial exploratory investigation, quantitative OCTA examination might be a useful first step in the development of artificial intelligencebased stratification of AMD patient subsets.

\section{ACKNOWLEDGEMENTS}

Funding. No funding or sponsorship was received for this study or publication of this article.

Authorship. All named authors meet the International Committee of Medical Journal Editors (ICMJE) criteria for authorship for this article, take responsibility for the integrity of the work as a whole, and have given their approval for this version to be published.

Author Contributions. Alessandro Arrigo MD and Alessia Amato MD (study design, data analysis, data interpretation, manuscript draft); Costanza Barresi MD, Emanuela Aragona MD, 
Andrea Saladino MD, Adelaide Pina OD and Francesca Calcagno MsC (data collection, data analysis, revision of the draft); Francesco Bandello MD, FEBO and Maurizio Battaglia Parodi MD,FEBO (data interpretation, revision of the draft, supervisors of the entire study).

Disclosures. Francesco Bandello consultant for: Alcon (Fort Worth, Texas, USA), Alimera Sciences (Alpharetta, Georgia, USA), Allergan Inc (Irvine, California, USA), Farmila-Thea (Clermont-Ferrand, France), Bayer SheringPharma (Berlin, Germany), Bausch And Lomb (Rochester, New York, USA), Genentech (San Francisco, California, USA), Hoffmann-LaRoche (Basel, Switzerland), NovagaliPharma (Évry, France), Novartis (Basel, Switzerland), Sanofi-Aventis (Paris, France), Thrombogenics (Heverlee, Belgium), Zeiss (Dublin, USA). All other authors have no disclosures to declare.

Compliance with Ethics Guidelines. The study was approved by the ethical committee of IRCCS Scientific Institute San Raffaele Hospital (protocol ID: MIRD), which verified its conduction in accordance with the Declaration of Helsinki.

Data Availability. The datasets generated during and/or analyzed during the current study are available from the corresponding author on reasonable request.

Open Access. This article is licensed under a Creative Commons Attribution-NonCommercial 4.0 International License, which permits any non-commercial use, sharing, adaptation, distribution and reproduction in any medium or format, as long as you give appropriate credit to the original author(s) and the source, provide a link to the Creative Commons licence, and indicate if changes were made. The images or other third party material in this article are included in the article's Creative Commons licence, unless indicated otherwise in a credit line to the material. If material is not included in the article's Creative Commons licence and your intended use is not permitted by statutory regulation or exceeds the permitted use, you will need to obtain permission directly from the copyright holder. To view a copy of this licence, visit http://creativecommons.org/licenses/by$\mathrm{nc} / 4.0 /$.

\section{REFERENCES}

1. Wong TY, Chakravarthy U, Klein R, Mitchell $\mathrm{P}$, Zlateva G, Buggage R, Fahrbach K, Probst C, Sledge I. The natural history and prognosis of neovascular age-related macular degeneration: a systematic review of the literature and meta-analysis. Ophthalmology. 2008;115(1):116-26.

2. Farazdaghi MK, Ebrahimi KB. Role of the choroid in age-related macular degeneration: a current review. J Ophthalmic Vis Res. 2019;14(1):78-87.

3. Campochiaro PA. Molecular pathogenesis of retinal and choroidal vascular diseases. Prog Retin Eye Res. 2015;49:67-81.

4. Lindner $\mathrm{M}$, Bezatis A, Czauderna J, Becker E, Brinkmann CK, Schmitz-Valckenberg S, Fimmers R, Holz FG, Fleckenstein M. Choroidal thickness in geographic atrophy secondary to age-related macular degeneration. Invest Ophthalmol Vis Sci. 2015;56(2):875-82.

5. Thorell MR, Goldhardt R, Nunes RP, de Amorim Garcia Filho CA, Abbey AM, Kuriyan AE, Modi YS, Gregori G, Yehoshua Z, Feuer W, Sadda S, Rosenfeld PJ. Association between subfoveal choroidal thickness, reticular pseudodrusen, and geographic atrophy in age-related macular degeneration. Ophthalm Surg Lasers Imag Retina. 2015;46(5): 513-21.

6. Sohn EH, Flamme-Wiese MJ, Whitmore SS, Workalemahu G, Marneros AG, Boese EA, Kwon YH, Wang K, Abramoff MD, Tucker BA, Stone EM, Mullins RF. Choriocapillaris degeneration in geographic atrophy. Am J Pathol. 2019;189(7): 1473-80.

7. Nassisi M, Baghdasaryan E, Borrelli E, Ip M, Sadda SR. Choriocapillaris flow impairment surrounding geographic atrophy correlates with disease progression. PLoS One. 2019;14(2): e0212563.

8. Spaide RF, Jaffe GJ, Sarraf D, Freund KB, Sadda SR, Staurenghi G, Waheed NK, Chakravarthy U, Rosenfeld PJ, Holz FG, Souied EH, Cohen SY, Querques G, Ohno-Matsui K, Boyer D, Gaudric A, Blodi B, Baumal CR, Li X, Coscas GJ, Brucker A, Singerman L, Luthert P, Schmitz-Valckenberg S, Schmidt-Erfurth U, Grossniklaus HE, Wilson DJ, Guymer R, Yannuzzi LA, Chew EY, Csaky K, Monés JM, Pauleikhoff D, Tadayoni R, Fujimoto J. 
Consensus nomenclature for reporting neovascular age-related macular degeneration data: consensus on neovascular age-related macular degeneration nomenclature study group. Ophthalmology. 2020;127(5):616-36.

9. Schindelin J, Arganda-Carreras I, Frise E, Kaynig V, Longair M, Pietzsch T, Preibisch S, Rueden C, Saalfeld S, Schmid B, Tinevez JY, White DJ, Hartenstein V, Eliceiri K, Tomancak P, Cardona A. Fiji: an opensource platform for biological-image analysis. Nat Methods. 2012;9(7):676-82.

10. Parodi MB, Arrigo A, Calamuneri A, Aragona E, Bandello F. Multimodal imaging in subclinical best vitelliform macular dystrophy. Br J Ophthalmol. 2020. https://doi.org/10.1136/bjophthalmol-2020317635 .

11. Spaide RF, Curcio CA. Drusen characterization with multimodal imaging. Retina. 2010;30(9):1441-54.

12. Invernizzi A, Benatti E, Cozzi M, Erba S, Vaishnavi S, Vupparaboina KK, Staurenghi G, Chhablani J, Gillies M, Viola F. Choroidal structural changes correlate with neovascular activity in neovascular age related macular degeneration. Invest Ophthalmol Vis Sci. 2018;59(10):3836-41.
13. Nassisi M, Fan W, Shi Y, Lei J, Borrelli E, Ip M, Sadda SR. Quantity of intraretinal hyperreflective foci in patients with intermediate age-related macular degeneration correlates with 1-year progression. Invest Ophthalmol Vis Sci. 2018;59(8):3431-9.

14. Parodi MB, Arrigo A, Romano F, Aragona E, Marchese A, Cicinelli MV, Mercuri S, Bandello F. Hyperreflective foci number correlates with choroidal neovascularization activity in angioid streaks. Invest Ophthalmol Vis Sci. 2018;59(8):3314-9.

15. Romano F, Mercuri S, Arrigo A, Marchese A, Cicinelli MV, Albertini GC, Bandello F, Battaglia Parodi M. Identification of hyperreflective foci in angioid streaks. Eye (Lond). 2019;33(12):1916-23.

16. Bhutto I, Lutty G. Understanding age-related macular degeneration (AMD): relationships between the photoreceptor/retinal pigment epithelium/ Bruch's membrane/choriocapillaris complex. Mol Aspects Med. 2012;33(4):295-317.

17. Spaide RF, Fujimoto JG, Waheed NK, Sadda SR, Staurenghi G. Optical coherence tomography angiography. Prog Retin Eye Res. 2018;64:1-55. 